\title{
Velocity-space sensitivity of the time-of-flight neutron spectrometer at JET
}

Jacobsen, Asger Schou; Salewski, Mirko; Eriksson, J.; Ericsson, G.; Hjalmarsson, A.; Korsholm, Søren Bang; Leipold, Frank; Nielsen, Stefan Kragh; Rasmussen, Jesper; Stejner Pedersen, Morten

Published in:

Review of Scientific Instruments

Link to article, DOI:

$10.1063 / 1.4885477$

Publication date:

2014

Link back to DTU Orbit

Citation (APA):

Jacobsen, A. S., Salewski, M., Eriksson, J., Ericsson, G., Hjalmarsson, A., Korsholm, S. B., Leipold, F., Nielsen, S. K., Rasmussen, J., \& Stejner Pedersen, M. (2014). Velocity-space sensitivity of the time-of-flight neutron spectrometer at JET. Review of Scientific Instruments, 85, [11E103]. https://doi.org/10.1063/1.4885477

\section{General rights}

Copyright and moral rights for the publications made accessible in the public portal are retained by the authors and/or other copyright owners and it is a condition of accessing publications that users recognise and abide by the legal requirements associated with these rights.

- Users may download and print one copy of any publication from the public portal for the purpose of private study or research.

- You may not further distribute the material or use it for any profit-making activity or commercial gain

- You may freely distribute the URL identifying the publication in the public portal 


\section{Velocity-space sensitivity of the time-of-flight neutron spectrometer at JET ${ }^{\mathrm{a}}$}

A.S. Jacobsen, ${ }^{2}$, b) M. Salewski, ${ }^{2}$ J. Eriksson, ${ }^{3}$ G. Ericsson, ${ }^{3}$ A. Hjalmarsson, ${ }^{3}$ S.B. Korsholm, ${ }^{2}$ F. Leipold, ${ }^{2}$ S.K. Nielsen, ${ }^{2}$ J. Rasmussen, ${ }^{2}$ M. Stejner, ${ }^{2}$ and JET EFDA contributors ${ }^{1, c)}$

1) JET-EFDA, Culham Science Centre, Abingdon, OX14 $3 \mathrm{DB}, \mathrm{UK}$

${ }^{2)}$ Association Euratom - DTU, Technical University of Denmark, Department of Physics, Kgs. Lyngby, Denmark

3) Association Euratom - VR, Uppsala University, Department of Physics and Astronomy, Uppsala, Sweden

(Dated: 4 February 2015)

The velocity-space sensitivities of fast-ion diagnostics are often described by so-called weight functions. Recently, we formulated weight functions showing the velocity-space sensitivity of the often dominant beamtarget part of neutron energy spectra. These weight functions for neutron emission spectrometry (NES) are independent of the particular NES diagnostic. Here we apply these NES weight functions to the time-of-flight spectrometer TOFOR at JET. By taking the instrumental response function of TOFOR into account, we calculate time-of-flight NES weight functions that enable us to directly determine the velocity-space sensitivity of a given part of a measured time-of-flight spectrum from TOFOR.

\section{INTRODUCTION}

The detection and analysis of fast ions in fusion devices are important since fusion-born alpha-particles are foreseen to contribute a large part of the heating in an eventual fusion power plant. Fast ions can be diagnosed using a variety of different diagnostics. One such diagnostic is neutron emission spectrometry (NES) in which the energy spectrum of neutrons produced in fusion reactions is measured. The energies of these neutrons depend on the energy released in the fusion reaction and on the energies of the reacting ions. Thus, by analysing the neutron energy spectrum, information on velocities of fast ions in the plasma can be gained. We recently derived weight functions describing the velocity-space sensitivities of the often dominant beam-target part of neutron energy spectra. In particular, they show the observable regions in velocity space and, for a given fast-ion velocity distribution function, the regions where most neutrons are generated. Here we apply these previously derived general expressions for neutron energy spectra to the time-of-flight spectrometer TOFOR at JET by taking the instrumental response function of TOFOR into account.

In section II the TOFOR instrument is described. The NES weight functions are introduced in section III, and in section IV we calculate time-of-flight NES weight functions for TOFOR and show examples of their applications. The results are summarized in section $\mathrm{V}$.

\footnotetext{
a) Contributed paper published as part of the Proceedings of the 20th Topical Conference on High-Temperature Plasma Diagnostics, Atlanta, Georgia, June, 2014

b) Author to whom correspondence should be addressed: Ajsen@fysik.dtu.dk

c) See the appendix of F. Romanelli, et al., Proceedings of the 24th IAEA Fusion Energy Conference 2012, San Diego, USA.
}

\section{NEUTRON TIME-OF-FLIGHT SPECTROMETER TOFOR}

TOFOR $^{1}$ is a time-of-flight spectrometer. It measures the time it takes for neutrons to travel between two sets of plastic scintillator detectors. The time-of-flight depends on the incident neutron energy; $2.45 \mathrm{MeV}$ neutrons from the D-D reaction result in flight times centered around $65 \mathrm{~ns}$. TOFOR is located in the roof laboratory 19 metres above JET and has a vertical view of the plasma so that the line-of-sight of TOFOR is perpendicular to the magnetic field. The viewing cone of TOFOR covers a large portion of the plasma cross-section, including the plasma center.

\section{NES WEIGHT FUNCTIONS}

Weight functions determine the velocity-space sensitivity of a given diagnostic. Weight functions have previously been calculated for fast-ion $\mathrm{D}_{\alpha}$ spectroscopy $(\text { FIDA })^{2,3}$ and collective Thomson scattering (CTS $)^{4}$. Furthermore, weight functions were recently developed for the beam-target part of neutron energy spectra measured using $\mathrm{NES}^{5}$. NES weight functions relate a measurement in a given neutron energy range to the fast-ion distribution function:

$$
\begin{aligned}
& s\left(E_{n, 1}, E_{n, 2}, \phi\right)= \\
& \iiint w\left(E_{n, 1}, E_{n, 2}, \phi, E, p, \mathbf{r}\right) f(E, p, \mathbf{r}) \mathrm{d} E \mathrm{~d} p \mathrm{~d} \mathbf{r},
\end{aligned}
$$

where $s\left(E_{n, 1}, E_{n, 2}, \phi\right)$ is the rate of neutrons with energies between $E_{n, 1}$ and $E_{n, 2}$ measured with an instrument with a line-of-sight at a projection angle, $\phi$, to the magnetic field. $w\left(E_{n, 1}, E_{n, 2}, \phi, E, p, \mathbf{r}\right)$ is the weight function calculated for specific neutron energies and projection angle $\phi$ as a function of energy, $E$, and pitch, $p=\frac{v_{\|}}{v}$, of the fast ions. The weight function has a spatial dependence since it depends on the bulk ion temperature 


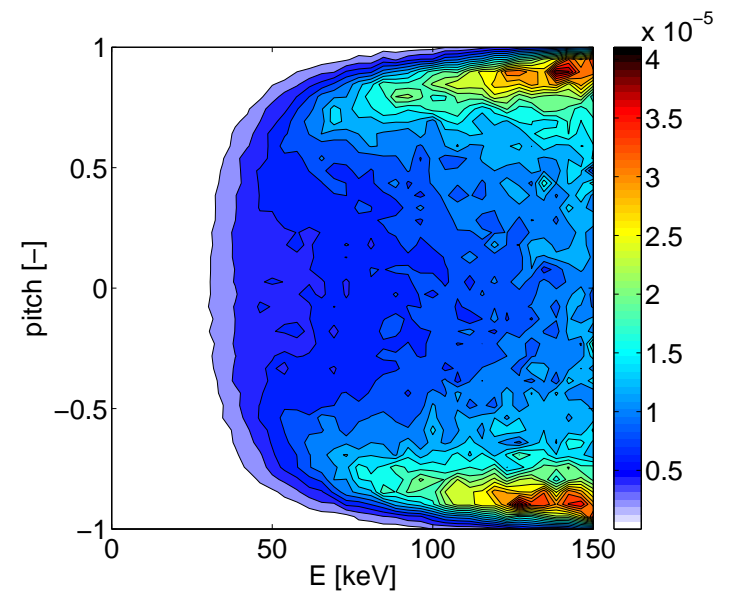

FIG. 1. NES weight function in units of [neutrons pr second pr fast ion] for a neutron energy of $2.6 \mathrm{MeV} \pm 0.015 \mathrm{MeV}$, calculated using a Monte Carlo approach.

and density, which can vary significantly along the lineof-sight. $f(E, p, \mathbf{r})$ is the fast-ion distribution function. NES weight functions are general and relate the fast-ion distribution function to a given neutron energy spectrum, irrespective of the specific instrument or technology used to acquire it. An example of a NES weight function is shown in figure 1 for a neutron energy of $2.6 \mathrm{MeV} \pm$ $0.015 \mathrm{MeV}$. The coloured region of the figure illustrates the part of $(E, p)$-space accessible to the specific neutron energy range, and the white region are inaccessible for this neutron energy range. A thermal ion temperature of $2.3 \mathrm{keV}$ has been used in these calculations.

\section{TIME-OF-FLIGHT NES WEIGHT FUNCTIONS}

TOFOR measures time-of-flight spectra from which the neutron energy spectra must be inferred. It is therefore useful to derive weight functions directly relating the time-of-flight spectra to velocity-space. This transformation requires knowledge of the particular instrumental response function. The instrumental response function of TOFOR describes the time-of-flight signal produced for an incoming beam of neutrons. It is dominated by the flight-time of neutrons that scatter only once in both sets of detectors. However, multiple-scattering events as well as the finite sizes of the detectors broaden the spectra. Thus, a mono-energetic flux of neutrons will produce a distribution of flight times. The response of TOFOR to incident neutrons of various energies has been modeled in detail with Monte Carlo calculations, taking the full 3D geometry of the instrument into account ${ }^{1}$. The resulting response function is shown in figure 2. It transforms energy spectra to time-of-flight spectra so as to compare these with measurements by TOFOR.

Figure 3 shows a typical time-of-flight spectrum measured during JET discharge \#68138 as well as a spectrum

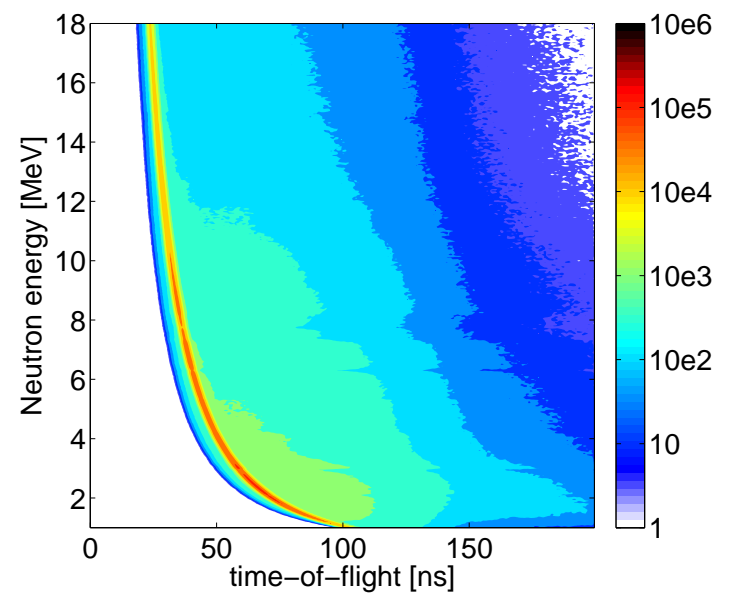

FIG. 2. TOFOR response function.

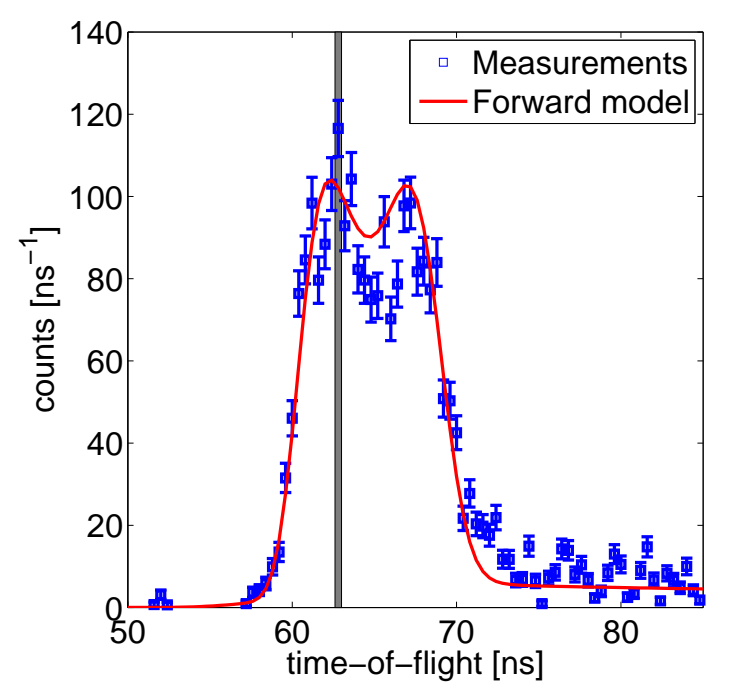

FIG. 3. Measured time-of-flight spectrum from JET discharge \#68138 compared with a calculated spectrum from a forward model. We show the velocity-space sensitivity of the shaded region in figure 4 .

calculated using a forward model. A double-hump resulting from the gyromotion of the fast ions appears in the spectrum at a flight-time of about $65 \mathrm{~ns}$ corresponding to a neutron energy of $2.45 \mathrm{MeV}$. The synthetic spectrum is calculated using fast-ion velocity distribution functions in the grid cells in the viewing cone simulated using TRANSP/NUBEAM ${ }^{6}$. The synthetic spectrum agrees reasonably well with the measured spectrum.

Figure 4 shows an example of a time-of-flight NES weight function for the time-of-flight range illustrated by the shaded region in figure 3. Time-of-flight NES weight functions are calculated from general NES weight functions and the TOFOR response functions according to

$$
w_{\text {tof }, i j l}=R_{n, k l} w_{\mathrm{E}_{\mathrm{n}}, i j k}
$$

where $R_{n}$ is the response function, $i, j, k$ and $l$ are indices 


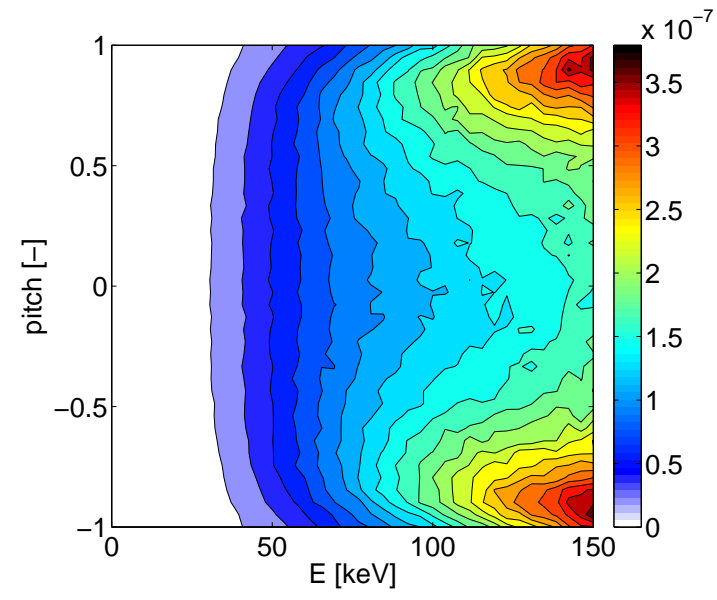

FIG. 4. Time-of-flight weight function in units of [neutrons pr second pr fast ion] for a flight-time of $62.8 \pm 0.2 \mathrm{~ns}$. This flight-time range is illustrated in figure 3 .

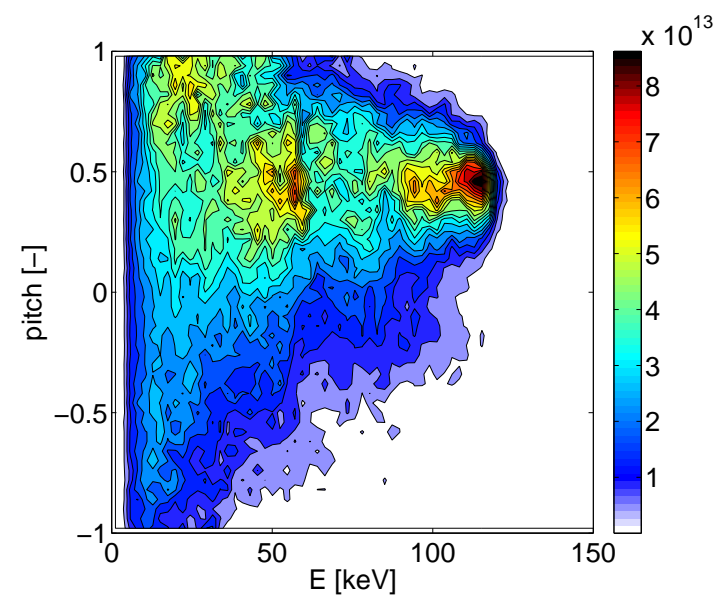

FIG. 5. Central fast-ion distribution function in units of [fast ions pr keV] calculated using TRANSP/NUBEAM.

of fast ion energy and pitch, neutron energy and time-offlight respectively. Repeated indices imply summation.

Figure 5 shows a central fast-ion distribution function from JET discharge \#68138 simulated using TRANSP/NUBEAM. TOFOR measures neutrons originating from the entire viewing cone. However, the majority will often come from the plasma center where both the thermal and fast ion densities are largest.

Figure 6 shows the product of the weight function in figure 4 and the central fast-ion distribution function in figure 5. This shows the part of the given central distribution function producing most of the detected neutrons. Since the measured spectrum agrees well with the synthetic spectrum (figure 3), the simulated fast-ion velocity distribution function should be a good model for the fast ion population in the plasma. Hence the predicted regions of maximum beam-target neutron production shown in figure 6 should be accurate. The main part of the signal in this flight-time range is caused by the ions

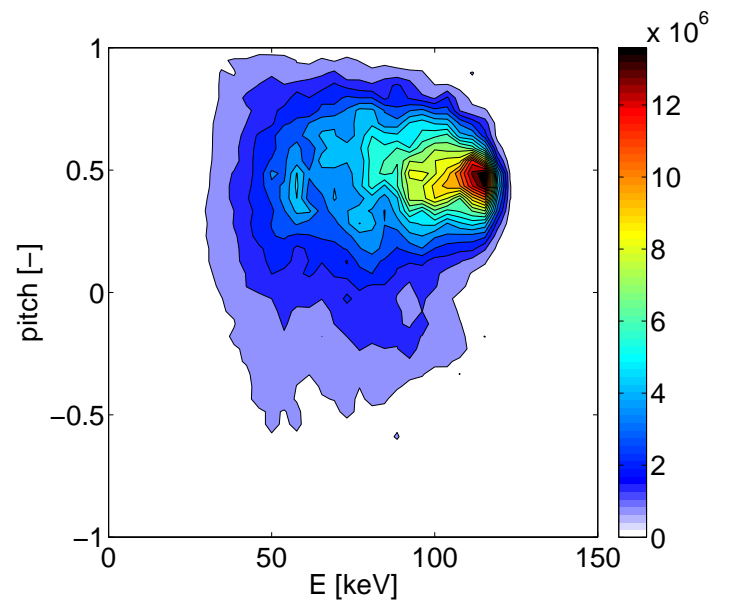

FIG. 6. Product of time-of-flight NES weight function from figure 4 and the fast-ion distribution function from figure 5.

with energies around $100 \mathrm{keV}$ and pitches of about 0.5 .

\section{v. CONCLUSIONS}

We have developed time-of-flight NES weight functions that illustrate the regions in velocity or energy-pitch space accessible to a given part of a measured time-offlight spectrum from TOFOR taking the response function into account. This allows us to directly determine the velocity-space sensitivity of given flight-time ranges in a TOFOR spectrum. This has several potential applications: as shown in figure 4, we can directly determine the velocity-space sensitivity and observable regions of a given part of a measured spectrum. Given a fast-ion distribution function, we can determine which part of the distribution function contributes most to the spectrum as shown in figure 6. Furthermore, they can be used in an efficient forward model by solving equation (1) for a given distribution function. Finally, weight functions on the form presented here can potentially enable us to use the NES diagnostic in velocity-space tomography to infer the fast-ion distribution function ${ }^{7,8}$.

\section{ACKNOWLEDGEMENTS}

This work, supported by the European Communities under the contract of Association between Euratom and DTU, was supported by EURATOM and carried out within the framework of the European Fusion Development Agreement. The views and opinions expressed herein do not necessarily reflect those of the European Commission.

\footnotetext{
${ }^{1}$ M. Gatu Johnson, et al. Nuclear Instruments and Methods in Physics Research Section A: Accelerators, Spectrometers, Detectors and Associated Equipment 591, 417 (2008).
} 
${ }^{2}$ W. W. Heidbrink, Y. Luo, K. H. Burrell, R. W. Harvey, R. I. Pinsker, and E. Ruskov, Plasma Physics and Controlled Fusion 49, 1457 (2007).

${ }^{3}$ M. Salewski, et al. Submitted, title: On velocity-space sensitivity of fast-ion D-alpha spectroscopy.

${ }^{4}$ M. Salewski, et al. Nuclear Fusion 51, 083014 (2011).

${ }^{5}$ A. S. Jacobsen, M. Salewski, J. Eriksson, G. Ericsson, S. B. Korsholm, F. Leipold, S. K. Nielsen, J. Rasmussen, and M. Stejner,
In preparation, title: Velocity-space sensitivity of neutron spectrometry measurements.

${ }^{6}$ A. Pankin, D. McCune, R. Andre, G. Bateman, and A. Kritz, Computer Physics Communications 159, 157 (2004).

${ }^{7}$ M. Salewski, et al. Nuclear Fusion 53, 063019 (2013).

${ }^{8}$ M. Salewski, et al. Nuclear Fusion 54, 023005 (2014). 